\title{
Correction to: a-Synuclein in blood exosomes immunoprecipitated using neuronal and oligodendroglial markers distinguishes Parkinson's disease from multiple system atrophy
}

\author{
Suman Dutta ${ }^{1}$ Simon Hornung ${ }^{1,14}$. Adira Kruayatidee ${ }^{1} \cdot$ Katherine N. Maina $^{1} \cdot$ Irish del Rosario ${ }^{2} \cdot$ Kimberly C. Paul $^{2}$. \\ Darice Y. Wong ${ }^{1}$ - Aline Duarte Folle ${ }^{2}$. Daniela Markovic ${ }^{3}$. Jose-Alberto Palma ${ }^{4}$. Geidy E. Serrano ${ }^{5}$. \\ Charles H. Adler ${ }^{6}$. Susan L. Perlman ${ }^{1}$. Wayne W. Poon ${ }^{7}$. Un Jung Kang ${ }^{4} \cdot$ Roy N. Alcalay ${ }^{8}$. Miriam Sklerov ${ }^{9}$. \\ Karen H. Gylys ${ }^{10,12} \cdot$ Horacio Kaufmann ${ }^{4}$. Brent L. Fogel ${ }^{1,11,12}$ • Jeff M. Bronstein ${ }^{1,12} \cdot$ Beate Ritz $^{2,12} \cdot$ Gal Bitan $^{1,12,13}$ (D)
}

Published online: 24 May 2021

(c) Springer-Verlag GmbH Germany, part of Springer Nature 2021

\section{Correction to: Acta Neuropathologica https://doi.org/10.1007/s00401-021-02324-0}

In the original publication, electronic supplementary material was missed to include during article processing stage and this is added to the erratum.

Supplementary Information The online version contains supplementary material available at https://doi.org/10.1007/s00401-021-02332-0.
Publisher's Note Springer Nature remains neutral with regard to jurisdictional claims in published maps and institutional affiliations.
The original article can be found online at https://doi.org/10.1007/ s00401-021-02324-0.

Gal Bitan

gbitan@mednet.ucla.edu

1 Department of Neurology, David Geffen School of Medicine, University of California, Los Angeles, CA 90095, USA

2 Department of Epidemiology, Fielding School of Public Health, University of California, Los Angeles, CA 90095, USA

3 Department of Medicine, Division of General Internal Medicine and Health Services Research, David Geffen School of Medicine, University of California, Los Angeles, CA 90095, USA

4 Department of Neurology, Dysautonomia Center, The Marlene and Paolo Fresco Institute for Parkinson's and Movement Disorders, New York University School of Medicine, New York, NY 10016, USA

5 Banner Sun Health Research Institute, Sun City, AZ 85351, USA

6 Mayo Clinic College of Medicine, Mayo Clinic Arizona, Scottsdale, AZ 85259, USA
7 Institute for Memory Impairments and Neurological Disorders, University of California, Irvine, CA 92697, USA

8 Department of Neurology, Taub Institute for Research on Alzheimer's Disease and the Aging Brain, Columbia University, New York, NY 10032, USA

9 Department of Neurology, University of North Carolina School of Medicine, Chapel Hill, NC 27599, USA

10 School of Nursing, University of California, Los Angeles, CA 90095, USA

11 Clinical Neurogenomics Research Center, David Geffen School of Medicine, University of California, Los Angeles, CA 90095, USA

12 Brain Research Institute, University of California, Los Angeles, CA 90095, USA

13 Molecular Biology Institute, University of California, Los Angeles, CA 90095, USA

14 Present Address: Division of Peptide Biochemistry, Technical University of Munich, 85354 Freising, Germany 